\title{
Edaravone, a Free Radical Scavenger, can Effect on the Inflammatory Biomarkers in Acute Ischemic Stroke Patients
}

Taizen Nakase*, Masahiro Sasaki and Akifumi Suzuki

Department of Stroke Science, Research Institute for Brain and Blood Vessels, Akita, Japan

*Corresponding author: Taizen Nakase, Department of Stroke Science, Research Institute for Brain and Blood Vessels, 6-10 Sensyu Kubota Machi, 010-0874, Akita, Japan, Tel: +81-18-833-0115, Fax: +81-18-833-2104; E-mail: nakase@akita-noken.jp

Rec date: May 19, 2014, Acc date: Jun 21, 2014, Pub date: Jun 24, 2014

Copyright: ( 2014 Nakase T, et al. This is an open-access article distributed under the terms of the Creative Commons Attribution License, which permits unrestricted use, distribution, and reproduction in any medium, provided the original author and source are credited.

\begin{abstract}
Background: This study was aimed to explore whether the free radical scavenger, edaravone, can reduce the inflammatory response providing better outcome along with not only conventional therapy but also recombinant tissue plasminogen activator (rt-PA) thrombolytic therapy.

Results: Acute ischemic stroke patients were enrolled ( $n=64$, average 73.3 year-old) for measuring interleukin- 6 (IL-6), tumor necrosis factor (TNFa) and malondialdehyde-modified LDL (MDA-LDL) as inflammatory biomarkers at 3 time points: before medication, $5 \mathrm{hrs}$ and $24 \mathrm{hs}$ following medication. All patients were classified into four groups depending on the medication: conventional therapy without edaravone $(n=15)$, conventional therapy with edaravone $(n=34)$, rt-PA thrombolytic therapy without edaravone $(n=4)$ and rt-PA thrombolytic therapy with edaravone $(n=11)$. Neurological alteration was assessed by NIH Stroke Scale on admission and at 1 month. As the results, the inflammatory markers were reduced in the edaravone treated group, although it was not significant. While, IL-6 was significantly increased at $24 \mathrm{hrs}$ in the thrombolytic group than the conventional group. Patients treated with rt-PA and edaravone exhibited better outcome than only rt-PA treated patients, although there was no difference of the recanalization rate.
\end{abstract}

Conclusion: Edaravone may be able to decrease the amplified inflammatory response following brain infarction, providing better outcome.

Keywords: Inflammatory biomarkers; Brain infarction; Acute therapy; Edaravone

\section{Introduction}

The inflammatory response is exacerbated at the acute phase of brain infarction. The amplified inflammation might relate to not only the worsening of ischemic lesion but also the poor outcome $[1,2]$. Especially, acute thrombolytic therapy by intravenous administration of recombinant tissue plasminogen activator (rt-PA) can cause the increase of inflammation [3,4]. Therefore, the reduction of inflammatory response following ischemia might be critical for the acute treatment of brain infarction. Up to the present, several studies have been reported the effect of statins regarding the reduction of inflammatory response $[5,6]$. Meanwhile, edaravone, a free radical scavenger, has been used for treatment of acute ischemic stroke patients in Japan since 2001. The effect of edaravone has been reported to reduce oxidative stress [7] and protecting neurovascular unit $[8,9]$, resulting in clinically better outcome [10]. Herein, this study was aimed to investigate how edaravone could reduce the inflammatory response in the acute brain infarction in the clinical situation.

\section{Methods}

\section{Patients}

Following the approval of the ethical committee of Research Institute for Brain and Blood Vessels -Akita (ref.\#09-10), acute ischemic stroke patients admitted to the hospital within $24 \mathrm{hrs}$ after the onset were consecutively asked to participate in this study between July 2009 and December 2010. Consented patients were enrolled in this study $(n=64,73.3 \pm 12.2$ y.o.). All patients were clinically diagnosed by magnetic resonance imaging (MRI: Sigma 1.5T, GE healthcare, Tokyo, Japan) using diffusion weighted images (DWI, TR: $5800 \mathrm{sec}, \mathrm{TE}: 76.2 \mathrm{sec}$ ) and T2 weighted images (T2WI, TR: $3600 \mathrm{sec}$, TE: $96 \mathrm{sec}$ ) on admission. Magnetic resonance angiography (MRA) images were constructed by the 3-demensional time-of-flight method and rendered using the maximum intensity projection method. Then, these patients were classified into cardiogenic embolism $(n=33)$, atherothrombotic infarction $(n=20)$ and lacunar infarction $(n=11)$ following the clinical examinations and records. According to the acute medication, patients were classified into the group of conventional therapy $(n=49)$ and the group of thrombolytic therapy $(n=15)$. For the conventional therapy, anti-platelet medicines such as clopidogrel (75 mg once a day) and cilostazol (100mg twice a day) or anti-coagulation medicine such as warfarin (optimal dose was set by PT-INR 2-3) were adopted to patients following the guideline [11]. For the thrombolytic therapy, rt-PA (alteplase) was intravenously administered within $3 \mathrm{hrs}$ after stroke onset $(0.6 \mathrm{mg} / \mathrm{kg}$ intravenous drip infusion taking $1 \mathrm{hr}$ following $10 \%$ bolus injection). For the neuroprotective therapy, edaravone (Radicut ${ }^{\oplus}$, Mitsubishi Tanabe Pharma Corp. Osaka, Japan) was administered with intravenous drip infusion ( $30 \mathrm{mg}$ twice a day). The decision of the treatment in each patient was performed following the standard guidelines [12]. Because edaravone has toxicity for kidney function, edaravone was administered to a patient unless who showed kidney dysfunction 
(Blood Urea Nitrogen $>30 \mathrm{mg} / \mathrm{dl}$ or Serum Creatinine $>1.5 \mathrm{mg} / \mathrm{dl}$ ) Therefore, in each medication group, we could classify all patients into four subgroups: 1) conventional therapy without edaravone, 2) conventional therapy with edaravone, 3 ) thrombolytic therapy without edaravone and 4) thrombolytic therapy with edaravone. Neurological deficits were measured by National Institute of Health Stroke Scale (NIHSS), and the alteration was assessed by comparing that of on admission and at 1 month later. Recanalization of the occluded artery by rt-PA administration was assessed on the findings of MRA performed on the second day. Hemorrhagic change of the ischemic lesion was assessed by the observations of follow-up computed brain tomography (Aquilion One, Toshiba Medical Systems, Tochigi, Japan). Clinical risk factors were collected from the patient's record. Criteria of risks are as following: hypertension $(>140 \mathrm{mmHg}$ of the systolic BP or $>90 \mathrm{mmHg}$ of the diastolic BP, or currently prescribed anti-hypertensive drugs), diabetes mellitus (spontaneous blood sugar level $>200 \mathrm{mg} / \mathrm{dL}$ or currently prescribed anti-diabetic medication), hyperlipidemia $(>220 \mathrm{mg} / \mathrm{dL}$ serum total cholesterol or $>150 \mathrm{mg} / \mathrm{dL}$ triglyceride, or currently prescribed anti-dyslipidemic medication), alcohol drinking ( $>180 \mathrm{ml}$ of sake per day) and smoking. Details of patients' background were presented in Table 1. Blood sampling for measuring the concentration of interleukin-6 (IL-6), tumor necrosis factor $\alpha \quad(\mathrm{TNF} \alpha)$ and malondialdehyde-modified low-density lipoprotein (MDA-LDL), which stands for oxidized LDL, were performed at 3 time points: before medication, $5 \mathrm{hrs}$ and $24 \mathrm{hrs}$ following medication started. Briefly describing the laboratory methods, serum amount of IL- 6 and MDA-LDL was measured by enzyme-linked immunosorbent assay (ELISA) method, and serum amount of TNFa was measured by chemiluminescent enzyme immunoassay (CLEIA) method. All blood examination for inflammatory biomarkers was performed by the external laboratory (SRL Inc. Tokyo, Japan).

\section{Statistical analysis}

Data are presented as mean \pm standard deviation (SD). Because the number of patients in the thrombolytic therapy without edaravone group was very small $(n=4)$, we could not adopt data into the statistical analysis. Clinical backgrounds were compared between the edaravone treated group and the no edaravone group by Pearson's $\chi 2$ test. A comparison of mean value of the NIHSS and the alteration of NIHSS between that of on admission and at 1 month was performed with factorial ANOVA post-hoc test, Scheffe method among three subgroups. The amount of serum IL- 6 was compared between the patients of all thrombolytic therapy and the patients of conventional therapy at the different time points by a nonparametric MannWhitney's U test. All statistical analysis was performed by JMP9 software (SAS Institute Inc., Cary, NC).

\section{Results}

Clinical backgrounds of all patients were shown in Table 1. Age was significantly younger in the edaravone treated group compared with the no edaravone group. There was no significant difference in the percentage of risk factors between the edaravone treated and the no edaravone groups. The distribution of stroke subtypes was not different between two groups.

Both the conventional and the thrombolytic therapy groups were subdivided into two groups according to whether edaravone was administered or not (Table 2). The thrombolytic therapy without edaravone group showed relatively higher age compared with other groups. NIHSS on admission was significantly increased in the thrombolytic therapy with edaravone group compared with the conventional groups $(\mathrm{p}<0.001)$. The thrombolytic therapy without edaravone group showed higher point of NIHSS at 1 month compared with other groups. Moreover, the reduction of NIHSS at one month was significantly higher in the thrombolytic therapy with edaravone group compared with the conventional group with and without edaravone ( $\mathrm{p}=0.014$ and 0.003 , respectively). The recanalization rate was slightly better in the thrombolytic therapy with edaravone compared with the thrombolytic therapy without edaravone group. The rate of the hemorrhagic transformation of the ischemic lesion was the same between the thrombolytic therapy with and without edaravone (4/4 cases and 10/11 cases, respectively). The hematoma at the cortical area was observed in 2 of 4 patients in the thrombolytic therapy without edaravone, while no patients exhibited the cortical hematoma in the thrombolytic therapy with edaravone group.

\begin{tabular}{|l|l|l|}
\hline Edaravone & Used & None \\
\hline $\mathrm{n}$ & 45 & 19 \\
\hline $\mathrm{m} / \mathrm{f}$ & $31 / 14$ & $13-$ Jun \\
\hline Age & $69.2 \pm 11.2$ & $78.2 \pm 10.8 \mp$ \\
\hline Subtype (\%) & & \\
\hline Embolic & $20(44.4)$ & $5(26.3)$ \\
\hline Atherothrombotic & $16(35.6)$ & $11(57.9)$ \\
\hline Lacunar & $9(20.0)$ & $3(15.8)$ \\
\hline rt-PA used (\%) & $11(24.4)$ & $4(21.1)$ \\
\hline Risks (\%) & & \\
\hline Hypertension & $31(68.9)$ & $16(84.2)$ \\
\hline Hyperlipidemia & $11(24.4)$ & $8(42.1)$ \\
\hline Diabetes Mellitus & $9(20.0)$ & $5(26.3)$ \\
\hline AF & $12(26.7)$ & $5(26.3)$ \\
\hline CKD & $2(4.4)$ & $2(10.5)$ \\
\hline Alcohol & $12(26.7)$ & $3(15.8)$ \\
\hline Smoking & $9(20.0)$ & $3(15.8)$ \\
\hline Past stroke history & $9(20.0)$ & $8(42.1)$ \\
\hline
\end{tabular}

Table 1: Background of all cases.

$\mathrm{m} / \mathrm{f}:$ male female ratio; age: average \pm standard deviation; embolic: embolic stroke; atherothrombotic: atherothrombotic infarction, lacunar: lacunar infarction, rt-PA: recombinant tissue plasminogen activator, Af: Atrial Fibrillation and CKD: Chronic Kidney Disease. ł: $\mathrm{p}<0.01$. 
Citation: Nakase T, Sasaki M, Suzuki A (2014) Edaravone, a Free Radical Scavenger, can Effect on the Inflammatory Biomarkers in Acute Ischemic Stroke Patients. J Neurol Disord 2: 167. doi:10.4172/2329-6895.1000167

Page 3 of 6

\begin{tabular}{|c|c|c|c|c|}
\hline \multirow{2}{*}{$\begin{array}{l}\text { Therapy } \\
\text { Edaravone }\end{array}$} & \multicolumn{2}{|c|}{ Conventional } & \multicolumn{2}{|c|}{ Thrombolytic } \\
\hline & $(-)$ & $(+)$ & $(-)$ & $(+)$ \\
\hline$n$ & 15 & 34 & 4 & 11 \\
\hline Age & $77.7 \pm 9.8$ & $70.8 \pm 11.6$ & $80.3 \pm 15.5$ & $64.3 \pm 8.5$ \\
\hline \multicolumn{5}{|l|}{ NIHSS } \\
\hline On admission & $5.4 \pm 6.5$ & $6.6 \pm 5.9$ & $19.8 \pm 9.9$ & $16.0 \pm 5.6 \ddagger$ \\
\hline At 1 month & $4.4 \pm 6.0$ & $3.4 \pm 4.4$ & $18.0 \pm 19.2$ & $7.5 \pm 5.5$ \\
\hline$\triangle \mathrm{NIHSS}$ & $1.0 \pm 5.9$ & $3.3 \pm 3.6$ & $1.8 \pm 17.3$ & $8.5 \pm 4.4^{* *}$ \\
\hline Recanalization (\%) & n.a. & n.a. & $2(50.0)$ & $8(72.7)$ \\
\hline
\end{tabular}

Table 2: Neurological alteration after each treatment

NIHSS: National Institute of Health Stroke Scale,

$\Delta$ NIHSS $=($ NIHSS on admission $)-($ NIHSS at 1 month $) . \neq: \mathrm{p}<0.001$, compared with the conventional groups, and ${ }^{* *}: \mathrm{p}=0.01$, compared with the conventional groups.

\section{Alteration of inflammatory markers}
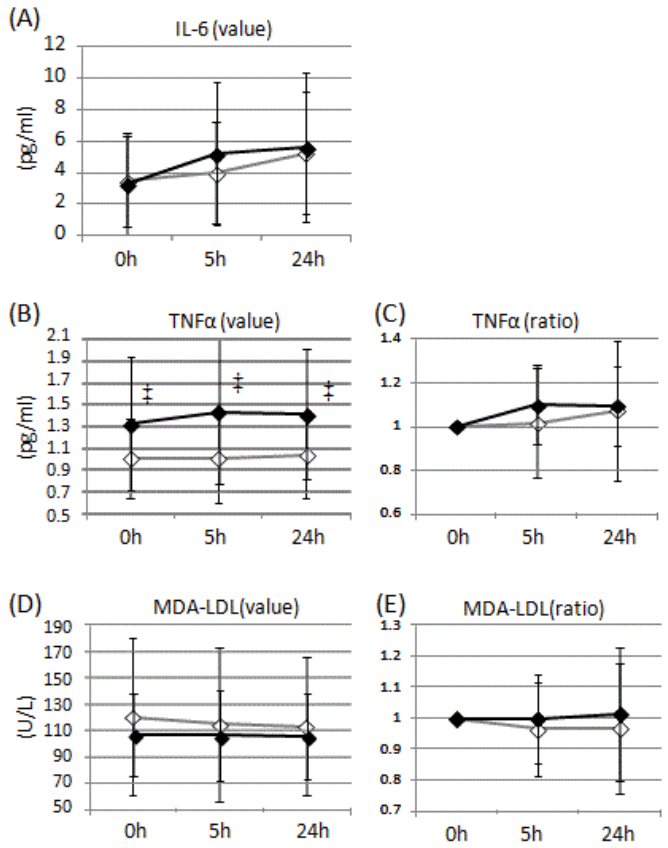

Figure 1: The alteration of inflammatory biomarkers (A: IL-6, B and $\mathrm{C}$ : TNFa, D and E: MDA-LDL) at different time points regarding with or without edaravone treatment.
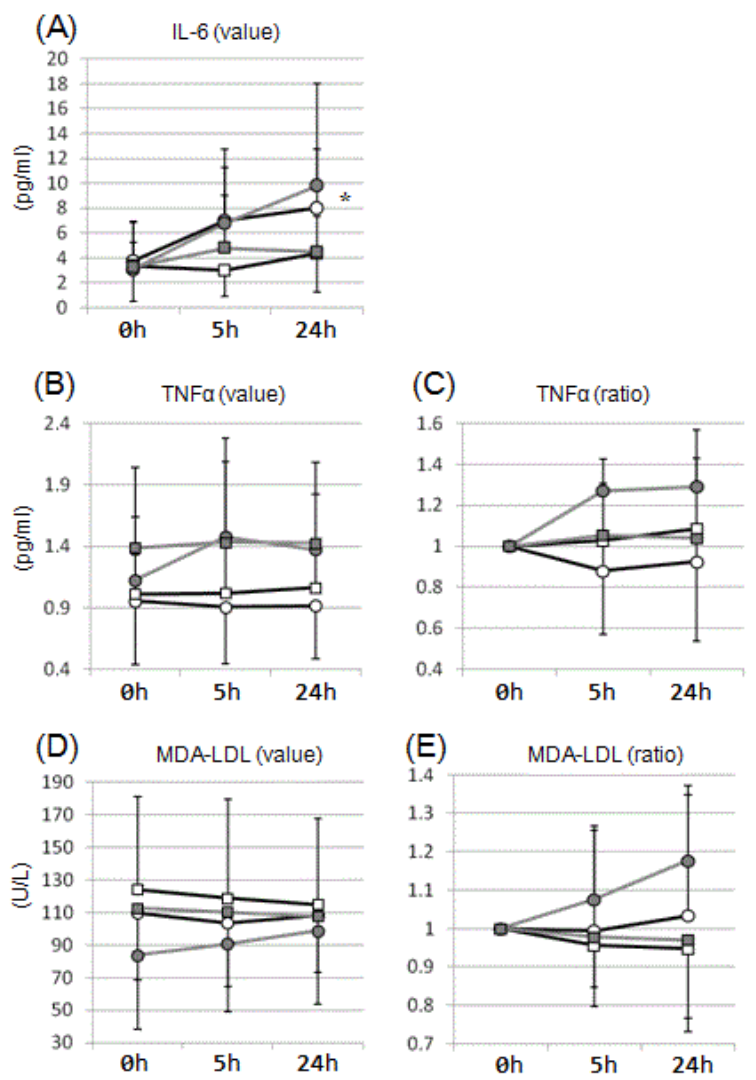

Figure 2

Figure 2: The alteration of inflammatory biomarkers (A: IL-6, B and $\mathrm{C}: \mathrm{TNF} \alpha, \mathrm{D}$ and $\mathrm{E}: \mathrm{MDA}-\mathrm{LDL}$ ) at different time points regarding 4 different therapy groups.

The results of inflammatory markers were shown in Figures 1 and 2. The edaravone treated group showed a slight reduction of IL- 6 and 
TNF $\alpha$ at 5 hrs following onset (Figure 1A and 1C). MDA-LDL was continuously decreased at $5 \mathrm{hrs}$ and $24 \mathrm{hrs}$ following onset (Figure 1E). The value of TNFa was significantly higher in the no edaravone group compared with the edaravone treated group (Figure 1B: $\mathrm{p}=0.001$ ). As shown in Figure 2A, although the level of IL-6 was already increased in the patients with thrombolytic therapy at $5 \mathrm{hrs}$ after medication started, the increase was not observed in the conventional therapy groups. At the time point of $24 \mathrm{hrs}$, the average amount of IL- 6 was significantly increased in the thrombolytic groups compared with the conventional groups (Figure 2A: $\mathrm{p}=0.008,9.02 \pm$ 5.57 and $4.44 \pm 3.10$, respectively).

The measured amount of TNFa presented a widely dispersed among four groups, suggesting the influence of stroke subtypes (Figure $2 \mathrm{~B}$ ). Thus far, the ratio of TNFa was referred for assessing the effect of medication. The conventional therapy did not alter the level of TNFa at both $5 \mathrm{hrs}$ and $24 \mathrm{hrs}$ regardless of edaravone administration. While the rt-PA administration amplified the amount of TNFa, the edaravone concurrent administration could decrease the TNFa level (Figure 2C).

As shown in Figure 2D, the measured amount of MDA-LDL was scattered among individual patients, however, in the calculation of MDA-LDL ratio, the thrombolytic therapy without edaravone group showed a continuous increase compared with other groups (Figure 2E).

Each line graph indicates the measured value of inflammatory biomarkers ( $\mathrm{A}, \mathrm{B}$ and $\mathrm{D})$ and the ratio against first time point $(\mathrm{C}$ and E). White diamond and black diamond show the edaravone administered group and the no edaravone group, respectively. (A) The edaravone administered group indicates slightly lower amount of IL-6 compared with the no edaravone group at $5 \mathrm{hrs}$. (B) The amount of TNFa is significantly higher in the no edaravone group compared with the edaravone administered group throughout the periods. (C) TNFa ratio is also decreased at $5 \mathrm{hrs}$ in the edaravone administered group. (E) MDA-LDL ratio is slightly decreased in the edaravone administered group compared with the no edaravone group at both 5 hrs and 24 hrs. $\ddagger$ : $\mathrm{p}<0.01$.

Each line graph indicates the measured value of inflammatory biomarkers (A, B and D) and the ratio against first time point $(\mathrm{C}$ and E). Gray circle, white circle, gray square and white square show the thrombolytic therapy without edaravone, the thrombolytic therapy with edaravone, the conventional therapy without edaravone and the conventional therapy with edaravone, respectively. (A) The thrombolytic groups indicate significantly higher amount of IL-6 compared with the conventional groups at $24 \mathrm{hrs}$ regardless of edaravone administration. ${ }^{*}: \mathrm{p}<0.05$, between the conventional groups and the thrombolytic groups. (C) Although the conventional therapy groups show no increase of TNFa ratio, the thrombolytic therapy without edaravone group shows the increase of TNFa ratio and the thrombolytic therapy with edaravone shows the decrease of TNFa ratio at both $5 \mathrm{hrs}$ and $24 \mathrm{hrs}$. (E) Only the thrombolytic therapy without edaravone group shows a continuous increase of MDA-LDL compared with other groups.

\section{Discussion}

In this study, we revealed that a free radical scavenger, edaravone, might be able to reduce the inflammatory response at the acute phase of brain infarction. Moreover, our data showed that the possibility in which the amplified inflammation by the rt-PA intravenous administration could be also decreased by edaravone.

Actually, the inflammation can be exacerbated by the ischemic cellular damage in the brain lesion. This inflammatory response at acute phase might not only influence on the expansion of ischemic lesion but also lead to the worse outcome $[1,2]$. Therefore, it can be said that the reduction of inflammation is a critical issue as the acute brain infarction therapy.

Statins have been interested in its effects in which the inflammatory response following ischemia can be reduced [5,6]. However, considering the effective dose and the time to achieve effective concentration, the statin administration has been still under debate regarding acute therapy of brain infarction $[13,14]$. Meanwhile, in Japan, edaravone has been officially available for clinical use for the treatment of acute brain infarction [10]. Edaravone was reported to decrease oxidative stress resulting in the reduction of inflammation $[7,15]$. Our previous study reported that the inflammatory response was restrained by edaravone administration in the acute phase of ischemic brain infarction, and the extent of gliosis was reduced at the chronic phase [16].

In this study, inflammatory markers of IL- 6 and TNFa were revealed to be increased at the acute phase of brain infarction. Moreover, edaravone was able to decrease this amplification at $5 \mathrm{hrs}$ after medication started, although it was not significant. While, the prominent increase of IL- 6 by rt-PA administration could not be reduced by edaravone. Moreover, the increase of MDA-LDL, which stands for the amplified oxidative stress, was observed only in the rtPA treated group. This oxidative stress might be also reduced by edaravone. The rt-PA expresses the thrombolytic efficacy by means of converting plasminogen to plasmin which could expedite the degradation of fibrin clot. However at the same time, plasmin has been reported to activate MMP3, resulting in the increase of the expression of MMP9 [17]. MMP9 has been reported to disrupt the basal membrane of the endothelial cells, causing decomposition of the blood brain barrier (BBB) $[18,19]$. Moreover, MMP9 was reported to increase the expression of cytokines such as granulocyte culturestimulating factor, and promote the activation of inflammatory cells [20]. Furthermore, previous reports suggested that rt-PA could directly raise inflammation by activating astrocytes and microglia $[21,22]$. According to the clinical studies, the better outcome following thrombolytic therapy might be related to the improvement of inflammatory response $[23,24]$. Thus far, although rt-PA is useful agent for thrombolytic therapy, it has a problematic issue in which inflammatory response will be exaggerated. Recently, experimental studies have revealed that edaravone could decrease the activation of MMP9 by restricting the expression of nuclear factor $(\mathrm{NF \kappa B})$ in the endotherial cells [25], and protect $\mathrm{BBB}$ in the ischemia-reperfusion injury $[8,26]$. Actually, our observation showed that hemorrhagic formation was not increased in the thrombolytic therapy with edaravone group. Rather, edaravone might be able to restrict hematoma formation. Moreover, edaravone has been reported to increase the rate of recanalization with rt-PA administration [27]. Oxidative stress induced by ischemia will cause the activation of plasminogen activator inhibitor-1 (PAI-1), resulting in the development of thrombosis [28]. Tumor necrosis factor (TNFa) has been reported to stimulate inflammatory cells for inducing various cytokines such as IL-1, platelet-activating factor (PAF) and PAI-1 [29]. It could be said that edaravone might be able to protect brain tissue 
against not only ischemic stress but also inflammatory response which was amplified by the recanalized blood flow by rt-PA.

This study was a prospective observational and not a randomized control study. Therefore, it is undeniable that patients who had not been administered edaravone might have some bias. Actually, edaravone was not adopted if a patient had kidney dysfunction. Moreover, the number of thrombolytic therapy without edaravone group was only 4 , and it was not able to perform the statistical analysis on this group. Therefore, since the number of patients in this study is also quite small, these findings have to be examined by a randomized control study with large number of cases. However, edaravone decreased the inflammatory response introduced by rt-PA and provide better recovery of neurological deficit. Therefore, it might be said that edaravone should be introduced at the same time as much as possible in case of acute brain infarction.

Meanwhile, according to the report from the pharmaceutical company (Mitsubishi Tanabe Pharma Corp.), edaravone should not be administered to the elderly patients who have complications of chronic renal dysfunction or dehydration. If edaravone is prescribed for two weeks complying with the informed document, the required clauses for avoiding side effects should be obeyed. Meanwhile, if we precisely looked at the report, such side effects were observed around 4th day after edaravone administrated. Nevertheless, we will propose that it might be acceptable to administrate edaravone at least only one time for reducing the inflammatory response following brain ischemia.

\section{Conclusion}

Even though inflammatory response was amplified following brain infarctioin, edaravone may be able to present the anti-inflammatory effects, providing better outcome. Further investigation will be intended to confirm our results.

\section{Acknowledgements}

We thank Ms. S. Kimura and Ms. M. Tobisawa for their excellent support in the statistical analysis.

\section{References}

1. Nakase T, Sasaki M, Yoshioka S, Ikeda Y, Suzuki A (2013) Risk of cognitive impairment in acute phase of intracerebral haemorrhage. Int J Stroke 8: E15.

2. Nakase T, Yamazaki T, Ogura N, Suzuki A, Nagata K (2008) The impact of inflammation on the pathogenesis and prognosis of ischemic stroke. J Neurol Sci 271: 104-109.

3. Baron JC (2005) How healthy is the acutely reperfused ischemic penumbra? Cerebrovasc Dis 20 Suppl 2: 25-31.

4. Shah IM, Macrae IM, Di Napoli M (2009) Neuroinflammation and neuroprotective strategies in acute ischaemic stroke - from bench to bedside. Curr Mol Med 9: 336-354.

5. StepieÅK, Tomaszewski M, Czuczwar SJ (2005) Neuroprotective properties of statins. Pharmacol Rep 57: 561-569.

6. Vaughan CJ, Delanty N (1999) Neuroprotective properties of statins in cerebral ischemia and stroke. Stroke 30: 1969-1973.

7. Zhang N, Komine-Kobayashi M, Tanaka R, Liu M, Mizuno Y, et al. (2005) Edaravone reduces early accumulation of oxidative products and sequential inflammatory responses after transient focal ischemia in mice brain. Stroke 36: 2220-2225.

8. Lee BJ, Egi Y, van Leyen K, Lo EH, Arai K (2010) Edaravone, a free radical scavenger, protects components of the neurovascular unit against oxidative stress in vitro. Brain Res 1307: 22-27.
9. Xi H, Akishita M, Nagai K, Yu W, Hasegawa H, et al. (2007) Potent free radical scavenger, edaravone, suppresses oxidative stress-induced endothelial damage and early atherosclerosis. Atherosclerosis 191: 281-289.

10. Edaravone Acute Infarction Study Group (2003) Effect of a novel free radical scavenger, edaravone (MCI-186), on acute brain infarction. Randomized, placebo-controlled, double-blind study at multicenters. Cerebrovasc Dis 15: 222-229.

11. Joint Committee on Guidlines for the Management of Stroke (2009) Ischemic Stroke and TIA. In: Japanese Guidelines for the Management of Stroke 2009: 53-56.

12. Joint Committee on Guidlines for the Management of Stroke (2009) Japanese Guidelines for the Management of Stroke 2009.

13. Baryan HK, Allan SM, Vail A, Smith CJ (2012) Systematic review and meta-analysis of the efficacy of statins in experimental stroke. Int J Stroke 7: 150-156.

14. Liu HL, Yang Y, Yang SL, Luo JP, Li H, et al. (2013) Administration of a loading dose of atorvastatin before percutaneous coronary intervention prevents inflammation and reduces myocardial injury in STEMI patients: a randomized clinical study. Clin Therapeut 35: 261-272.

15. Lapchak PA (2010) A critical assessment of edaravone acute ischemic stroke efficacy trials: is edaravone an effective neuroprotective therapy? Expert Opin Pharmacother 11: 1753-1763.

16. Nakase T, Yoshioka S, Suzuki A (2011) Free radical scavenger, edaravone, reduces the lesion size of lacunar infarction in human brain ischemic stroke. BMC Neurol 11: 39.

17. Candelario-Jalil E, Yang Y, Rosenberg GA (2009) Diverse roles of matrix metalloproteinases and tissue inhibitors of metalloproteinases in neuroinflammation and cerebral ischemia. Neuroscience 158: 983-994.

18. Aid S, Silva AC, Candelario-Jalil E, Choi SH, Rosenberg GA, et al. (2010) Cyclooxygenase- 1 and -2 differentially modulate lipopolysaccharideinduced blood-brain barrier disruption through matrix metalloproteinase activity. J Cereb Blood Flow Metab 30: 370-380.

19. Yepes M, Sandkvist M, Moore EG, Bugge TH, Strickland DK, et al. (2003) Tissue-type plasminogen activator induces opening of the bloodbrain barrier via the LDL receptor-related protein. J Clin Invest 112: 1533-1540.

20. Ohki M, Ohki Y, Ishihara M, Nishida C, Tashiro Y, et al (2010) Tissue type plasminogen activator regulates myeloid-cell dependent neoangiogenesis during tissue regeneration. Blood 115: 4302-4312.

21. Sheehan JJ, Tsirka SE (2005) Fibrin-modifying serine proteases thrombin, tPA, and plasmin in ischemic stroke: a review. Glia 50: 340-350.

22. Flavin MP, Zhao G, Ho LT (2000) Microglial tissue plasminogen activator (tPA) triggers neuronal apoptosis in vitro. Glia 29: 347-354.

23. Audebert HJ, Rott MM, Eck T, Haberl RL (2004) Systemic inflammatory response depends on initial stroke severity but is attenuated by successful thrombolysis. Stroke 35: 2128-2133.

24. Millán M, Sobrino T, Arenillas JF, Rodríguez-Yáñez M, García M, et al. (2008) Biological signatures of brain damage associated with high serum ferritin levels in patients with acute ischemic stroke and thrombolytic treatment. Dis Markers 25: 181-188.

25. Yagi K, Kitazato KT, Uno M, Tada Y, Kinouchi T, et al. (2009) Edaravone, a free radical scavenger, inhibits MMP-9-related brain hemorrhage in rats treated with tissue plasminogen activator. Stroke 40: 626-631.

26. Yamashita T, Kamiya T, Deguchi K, Inaba T, Zhang H, et al. (2009) Dissociation and protection of the neurovascular unit after thrombolysis and reperfusion in ischemic rat brain. J Cereb Blood Flow Metab 29: 715-725.

27. Kimura K, Aoki J, Sakamoto Y, Kobayashi K, Sakai K, et al. (2012) Administration of edaravone, a free radical scavenger, during t-PA infusion can enhance early recanalization in acute stroke patients--a preliminary study. J Neurol Sci 313: 132-136. 
Citation: Nakase T, Sasaki M, Suzuki A (2014) Edaravone, a Free Radical Scavenger, can Effect on the Inflammatory Biomarkers in Acute Ischemic Stroke Patients. J Neurol Disord 2: 167. doi:10.4172/2329-6895.1000167

Page 6 of 6

28. Dimova EY, Samoylenko A, Kietzmann T (2004) Oxidative stress and hypoxia: implications for plasminogen activator inhibitor-1 expression. Antioxid Redox Signal 6: 777-791.
29. Tuttolomondo A, Di Raimondo D, di Sciacca R, Pinto A, Licata G (2008) Inflammatory cytokines in acute ischemic stroke. Curr Pharm Des 14: 3574-3589. 